\section{Primary tumours of the optic nerve and its sheath}

\author{
Abstract \\ Purpose To describe the clinical, \\ neuroimaging, and pathologic features of \\ primary tumours of the optic nerve and its \\ sheath. \\ Methods Review of published cases and \\ personal series.
}

Results The most common primary tumour of the optic nerve is the benign glioma. This lowgrade astrocytoma usually can be followed without intervention. Progression of visual symptoms and signs may necessitate either surgery to remove the tumour or radiation therapy. The most common tumour of the optic nerve sheath is the meningioma. The optimum treatment for this lesion is stereotactic or threedimensional conformal fractionated radiation therapy, which generally results in stabilization or improvement in vision. A variety of other primary tumours may mimic, in both manifestations and imaging appearance, the more common glioma or meningioma. In such cases, the correct diagnosis may not be made until a biopsy is performed or the nerve is removed.

Conclusion Primary tumours of the optic nerve and its sheath are not uncommon. Diagnosis can often but not always be made by the results of a complete examination

Wilmer Eye Institute Johns Hopkins Hospital Baltimore, MD, USA

Correspondence: NR Miller Maumenee B-109 Johns Hopkins Hospital 600 North Wolfe Street Baltimore, MD 21287, USA Tel.: + 14109558679

Fax: + 14106149240

E-mail:nrmiller@

jhmi.edu

Received: 4 September 2003

Accepted: 4 September 2003 combined with imaging studies, particularly CT scanning and MR imaging. Management depends on the presumed or histologically verified nature of the tumour.

Eye (2004) 18, 1026-1037. doi:10.1038/sj.eye.6701592

Keywords: optic nerve; tumour; glioma; meningioma; radiation therapy

\section{Introduction}

Primary tumours of the optic nerve can be classified as intrinsic tumours and tumours of the sheath. In this paper, I will discuss both types of lesions.
NR Miller

Primary tumours of the optic nerve

The most common tumour of the optic nerve is the optic nerve glioma. Most of these tumours are benign but malignant gliomas of the optic nerve occur as do other tumours, including gangliogliomas, medulloepitheliomas, and vascular tumours, such as haemangioblastomas and haemangiopericytomas.

\section{Optic nerve glioma (benign)}

Optic nerve gliomas comprise about $1 \%$ of all intracranial tumours. ${ }^{1}$ They are almost always unilateral and occur more frequently in females than in males. These tumours may occur at any age, but most become symptomatic in childhood. Indeed, according to Chutorian et $a l^{2}$ $75 \%$ of patients with optic nerve gliomas become symptomatic in the first decade of life, and $90 \%$ become symptomatic during the first two decades of life. In a series of 33 patients with optic nerve gliomas reported by Rush et al, ${ }^{3}$ the age range was $2-46$ years, with a median age of 6.5 years and a mean age of 10.9 years. Optic nerve gliomas occurring in adults behave similarly to gliomas of the optic nerve in childhood.

Most cases of optic nerve glioma are sporadic, but several reports describe these tumours in siblings, and others describe them in various generations of several families. In all of these reports, the affected patients have had evidence of neurofibromatosis type 1 (NF1).

The symptoms and signs that occur in patients with optic nerve gliomas are well described, and include decreased visual function, proptosis (often associated with infradisplacement of the globe), optic disc swelling or pallor, and strabismus (Figure 1). ${ }^{4}$ Neither orbital nor ocular pain is typically present. Owing to chronic compression of the central retinal vein, some patients with optic nerve gliomas develop central retinal vein occlusion (CRVO), venous stasis retinopathy, optociliary shunt vessels, or rubeosis iridis with 
neovascular glaucoma. Others experience acute loss of vision, usually associated with development or worsening of proptosis, from haemorrhage into the tumour. ${ }^{5}$ However, not all optic nerve gliomas are symptomatic. Some are found during general screening of children with NF1. ${ }^{4}$ In many of these patients, visualevoked potentials (VEPs) are mildly abnormal.

A relationship between optic nerve glioma and NF1 is well established. ${ }^{6}$ The reported incidence of NF1 among patients with optic nerve or chiasmal gliomas ranges from 10 to $70 \%$ in large series, probably reflecting the differing degrees of thoroughness with which investigators examine their patients for the stigmata of NF1, different institutional biases, and patterns of

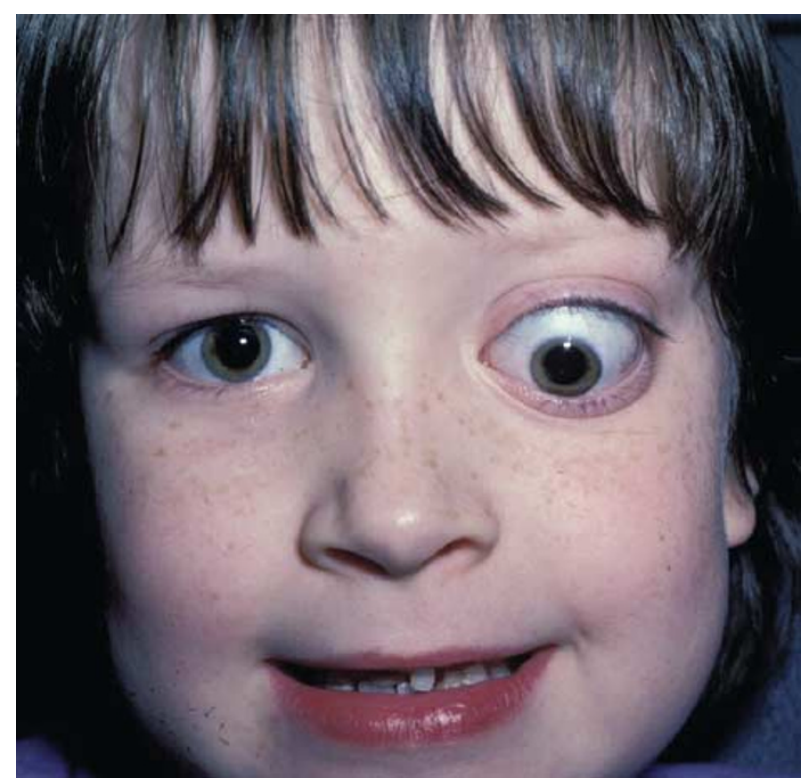

Figure 1 External appearance of a patient with an optic nerve glioma. referral. Conversely, the incidence of optic nerve glioma in patients with NF1 varies from 8 to $31 \%{ }^{6}$

The diagnosis of an optic nerve glioma can be confirmed by either computed tomographic (CT) scanning or magnetic resonance (MR) imaging. The appearance depends on whether or not the patient has NF1. In patients without NF1, there is almost always a fusiform enlargement of the optic nerve with a clearcut margin produced by the intact dural sheath (Figure 2a). In patients without NF1, the nerve is more irregular and tends to show both kinking and buckling as well as low-density areas within the nerve (Figure $2 b){ }^{7} \mathrm{MR}$ imaging typically shows gliomas to be hypo- to isointense on T1-weighted images and mildly to strongly hyperintense on proton density- and T2-weighted images. After intravenous injection of a paramagnetic substance such as gadolinium-DTPA, some gliomas enhance wholly or in part, and it is thought that such lesions are more metabolically active than those that do not enhance. ${ }^{8}$ MR imaging can also show extension of tumour and tumour-associated changes beyond the optic nerve into the chiasm, findings that may not be apparent on CT scanning (Figure 3). Although the appearance of some optic nerve gliomas may be mistaken for that of an optic nerve sheath meningioma, ${ }^{9}$ the distinction between the two entities usually is easy to make by combining clinical and imaging findings.

Some patients with optic nerve gliomas have an enlarged optic canal on the side of the lesion. The enlargement can be identified by both CT scanning and MR imaging. An enlarged optic canal does not indicate with certainty that the tumour extends intracranially, however. Arachnoid hyperplasia alone may be responsible for the enlargement. Conversely, a normalsized optic canal does not indicate that the tumour is confined within the orbit.
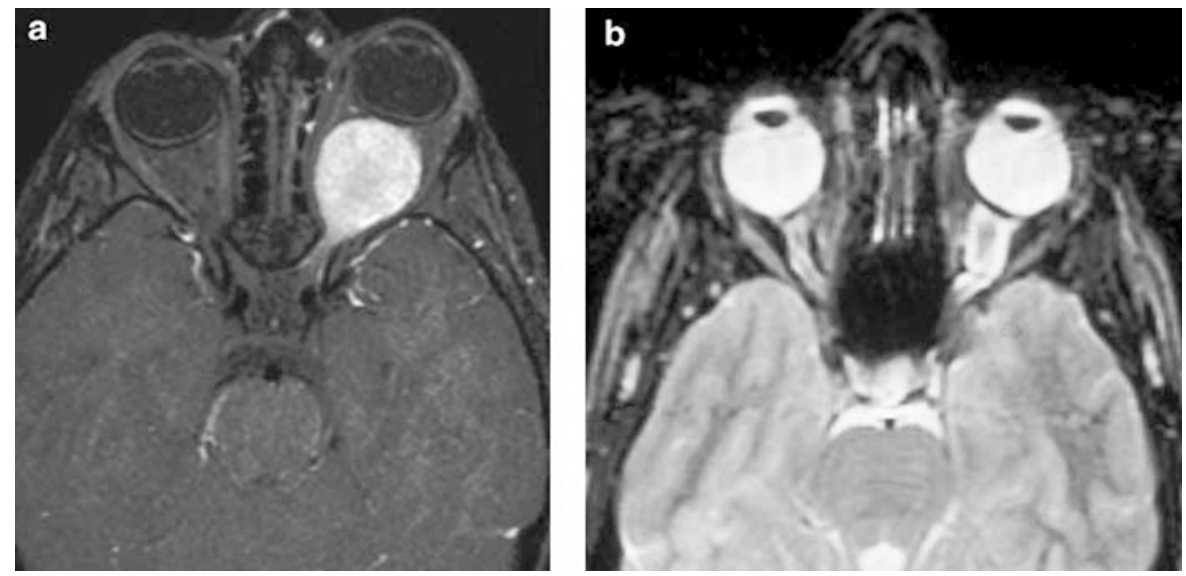

Figure 2 Imaging appearance of optic nerve gliomas: (a) in a patient without neurofibromatosis; (b) in a patient with neurofibromatosis. 


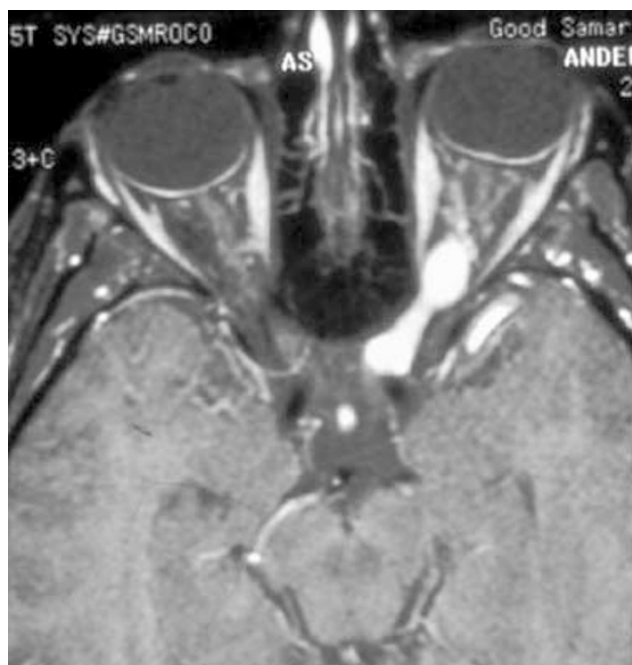

Figure 3 MR imaging of an optic nerve glioma showing intracranial extension of the tumour.

The gross appearance of an optic nerve glioma is characteristic. In patients without NF1, the process consists of a diffuse expansion of the nerve that may extend the entire length of the nerve or occur along any portion of it (Figure 4a). The expanded area may be solid or have areas with a gelatinous appearance. Haemorrhage may be present in some areas. In patients with NF1, the tumour not only expands the optic nerve parenchyma but often breaks through the pia-arachnoid to fill both the subarachnoid and subdural spaces (Figure $4 \mathrm{~b}$ ). ${ }^{10}$

However, the tumour almost always remains within the confines of the dural sheath of the optic nerve as long as it stays within the confines of the orbit or optic canal. Once it extends intracranially, however, it may remain primarily intraneural or develop a sizable exophytic component that in rare cases compresses the opposite optic nerve, optic chiasm, or both.

The natural history of optic nerve gliomas is almost always benign. ${ }^{4}$ Most grow slowly in a self-limited manner and some spontaneously regress. ${ }^{11}$ It is therefore not surprising that long-term studies indicate that patients who are not treated usually retain excellent, or at least have stable, visual function. Unfortunately, in most large series, the tumours have been excised rather than observed because of the concern that the tumour will extend to the optic chiasm and damage visual function in the opposite eye. In fact, this rarely occurs. Thus, although most investigators agree that complete removal of the tumour, whether by an orbital or a transcranial approach, is associated with excellent long-term survival, I agree with other authors ${ }^{12}$ that such treatment is rarely necessary and reserve removal of the optic nerve for patients with severe cosmetic disfigurement or imaging evidence of progressive extension of the tumour.
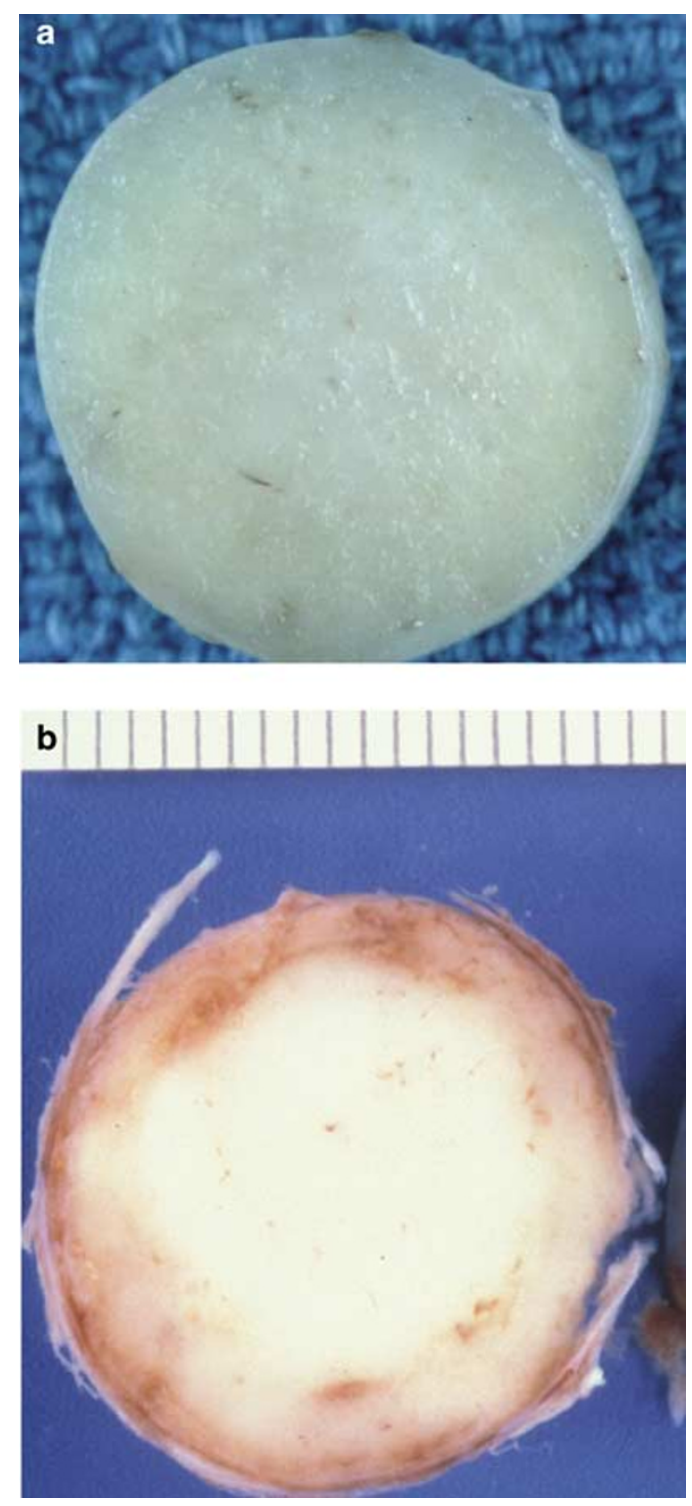

Figure 4 Macroscopic appearance of optic nerve gliomas: (a) in a patient without neurofibromatosis; (b) in a patient with neurofibromatosis.

The value of radiotherapy and chemotherapy in the treatment of optic nerve gliomas is even less clear than the value of surgery. ${ }^{12,13}$ Individual case reports indicate that some patients with presumed optic nerve gliomas experience improvement in vision and reduction in the size of the lesion following conventional fractionated radiation therapy or chemotherapy, but the results may not be any better than the natural history of the lesion, and, in addition, the treatment may produce a variety of complications.

There remain many unanswered questions pertaining to optic nerve gliomas: (a) What is the risk that the tumour will extend intracranially and place the patient at a higher risk of contralateral visual loss or death? (b) Are 
optic nerve gliomas that appear to grow really infiltrating a previously normal structure or have pre-existing tumour cells in the area entered a more aggressive growth phase? (c) Does the presence or absence of NF1 correlate with the behaviour of an optic nerve glioma? (d) What are the features of an optic nerve glioma that render it susceptible to radiotherapy, chemotherapy, or both? I believe that these questions and others can be answered only by following patients with gliomas confined to the optic nerve over extended periods with meticulous clinical examinations and MR imaging at regular intervals. In the meantime, I recommend that most patients with unilateral optic nerve gliomas, particularly those with NF1, be followed at regular intervals both clinically and with neuroimaging without intervention. Only if there is cosmetically unacceptable proptosis, progressive deterioration of visual function, evidence by MR imaging of definite tumour enlargement or extension but not to the optic chiasm, or a combination of these, should surgical excision of the lesion be considered.

In some cases, surgical excision of a glioma confined to the orbital portion of the optic nerve is best performed by a craniotomy to ensure removal of the entire tumour. In most cases, however, an orbital approach may suffice to remove the involved nerve, particularly when the main reason for surgery is cosmetically unacceptable proptosis. Regardless of the operation that is used, the involved eye need not be enucleated. An eye whose optic nerve is removed to treat an optic nerve glioma or meningioma (see below) usually does not become phthisical, particularly if care is taken to preserve the short posterior ciliary arteries, because there is an adequate collateral blood supply to the globe.

\section{Malignant optic nerve glioma}

Although most gliomas that involve the optic nerve have a benign histologic appearance and a relatively benign prognosis, malignant astrocytomas occasionally involve the anterior visual system, producing a clinical course of characterized by rapidly progressive visual loss, neurologic deficits, and, eventually, death. ${ }^{14}$ Unlike low-grade gliomas of the anterior visual system that occur in children, malignant gliomas almost always occur in adults. ${ }^{14}$ The age range is broad, ranging from the second to the eighth decade of life. Some reports suggested that these tumours occur primarily in men, whereas others report an equal incidence in men and women.

The specific pattern of visual loss that occurs in patients with malignant gliomas of the optic nerve appears to depend on the site of origin of the tumour. Tumours that originate in the proximal portion of the nerve produce a characteristic syndrome. ${ }^{14}$ The initial symptoms are monocular blurring of vision and retrobulbar pain simulating optic neuritis. The fundus of the affected eye initially may appear normal, but most patients rapidly develop evidence of occlusive vascular disease involving the optic disc, including venous stasis and oedema (Figure 5a). There may be extensive
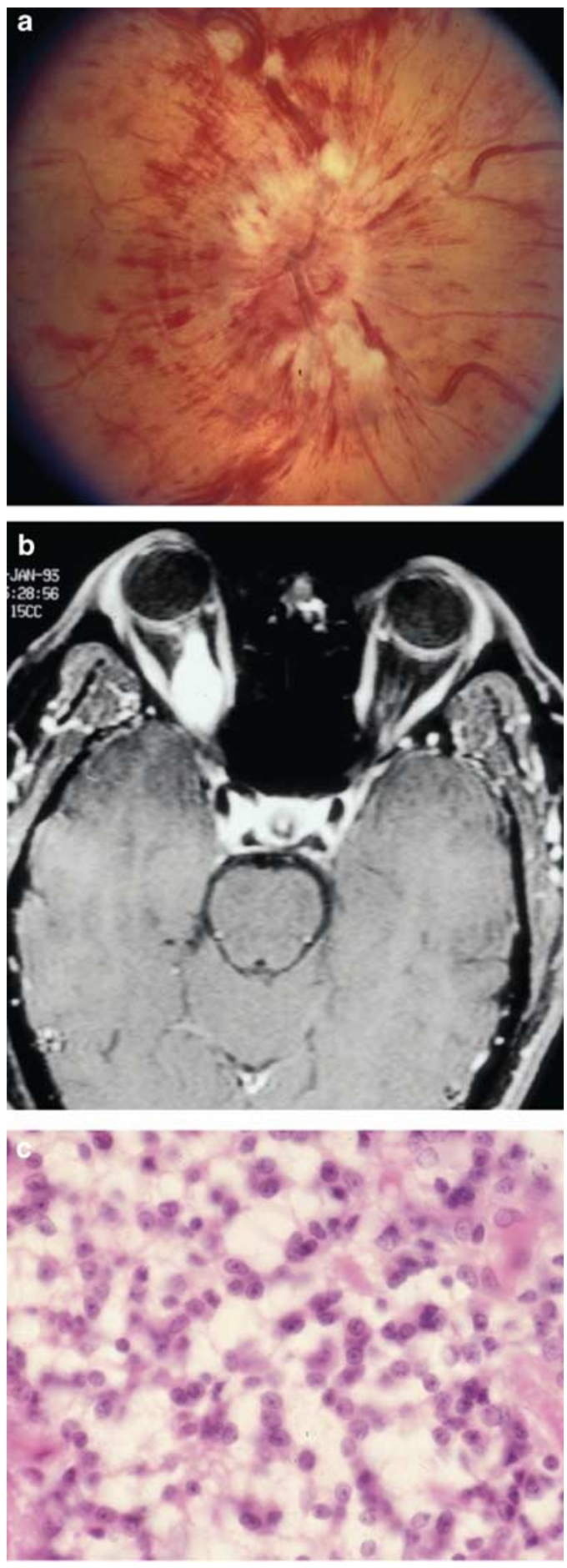

Figure 5 Malignant optic nerve glioma: (a) fundus showing appearance of a severe central retinal vein occlusion; (b) MR imaging of lesion; (c) histopathology. 
haemorrhage in the posterior pole, and the appearance of the fundus may thus resemble the ischaemic form of CRVO, and neovascular glaucoma may develop. This does not remain, however, a monocular disease. Within 5-6 weeks, both eyes become affected, and the patient soon becomes completely blind. Hypothalamic dysfunction, hemiparesis, and other neurologic deficits develop in the latter stages of the disease, and death usually occurs in less than 1 year. ${ }^{14}$

Malignant optic gliomas that originate in the distal portion of the optic nerve produce a similar syndrome of progressive unilateral visual loss, neurologic symptoms, and death; however, the visual loss in these patients is associated with a normal-appearing optic disc that eventually becomes pale. ${ }^{15}$

The neuroimaging appearance of a malignant glioma of the optic nerve is nonspecific. In some cases, the optic nerve appears diffusely thickened, and MR imaging after intravenous injection of a paramagnetic contrast agent may show marked enhancement of the nerve with inhomogeneity and cystic-appearing areas (Figure 5b). ${ }^{15,16}$

The pathologic features of a malignant optic nerve glioma are characteristic. ${ }^{14-16}$ The vascular and partially necrotic tumour occupies most of the nerve. In the orbit, it usually infiltrates the meninges of the nerve and the surrounding soft tissue, whereas intracranially, it eventually infiltrates the optic chiasm, hypothalamus, and adjacent parts of the brain. The histopathology of this tumour is completely different from that of the typical optic nerve glioma, being characterized by extreme cellular pleomorphism, nuclear hyperchromaticity, and scattered mitoses (Figure 5c). There are often numerous areas of vascular endothelial proliferation, necrosis, and haemorrhage, similar to those seen in glioblastomas.

There is no satisfactory treatment for a malignant optic nerve glioma, and death is the result in most cases. Shortterm success occasionally follows treatment with combined radiotherapy and chemotherapy.

\section{Ganglioglioma}

In rare instances, ganglion cell tumours originate within the substance of the optic nerve. ${ }^{17-19}$ Such a tumour may be thought to be an optic glioma until an exploration is performed and the nerve is removed or a biopsy of the lesion is obtained. The presentation of these tumours as well as their imaging characteristics are similar to those of optic nerve gliomas (Figure 6a), although the visual loss may be more rapidly progressive; however, their pathology is quite different. The tumour is composed of numerous ganglion cells, separated by connective tissue that is abundant in some areas and sparse in others. ${ }^{17-19}$
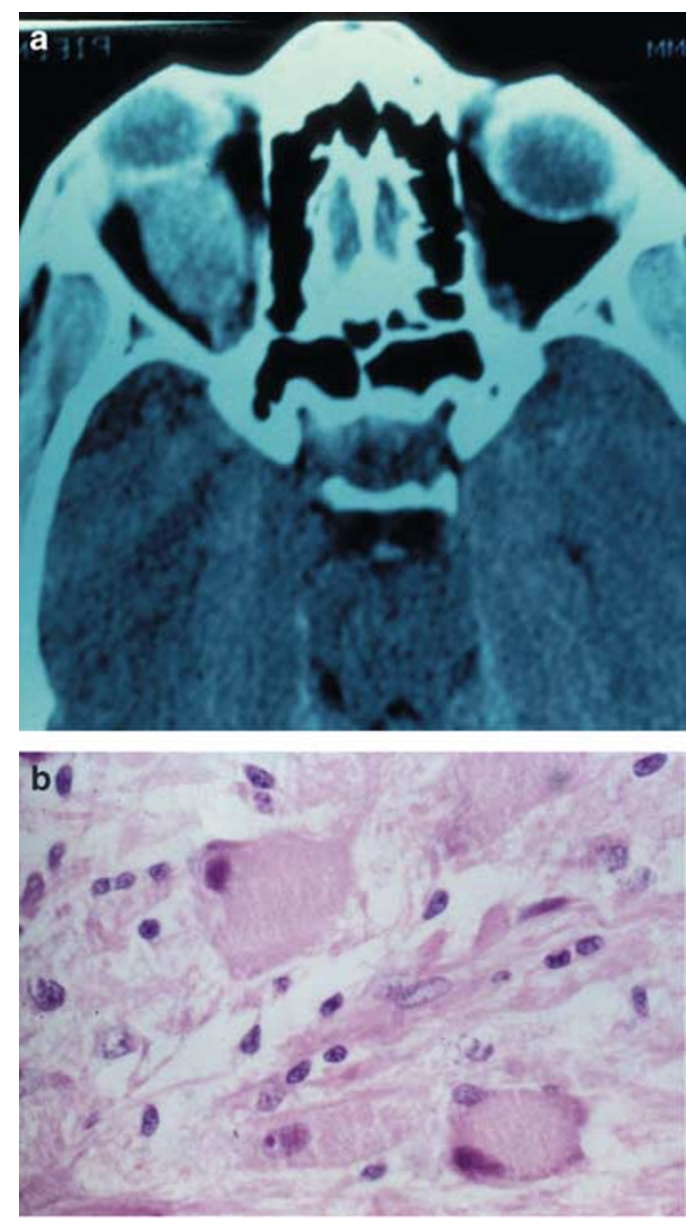

Figure 6 Ganglioglioma of the optic nerve: (a) MR appearance; (b) histopathology (Courtesy of Dr Clinton D McCord.)

Microscopic findings show neoplastic astrocytic nuclei throughout a hypercellular nerve associated with small numbers of randomly distributed, well-differentiated ganglionic elements as well as binucleate ganglion cells (Figure 6b). These tumours also show immunoreactivity to synaptophysin and neurofilament protein. ${ }^{19}$

\section{Medulloepitheliomas}

These tumours most commonly arise in the brain and spinal cord; however, they also may arise from the optic nerve. ${ }^{20-22}$ This is not surprising when one considers that the embryonic neuroepithelium that lines the invaginated optic vesicle is continuous with that lining the cavities of the optic nerve and the forebrain early in embryonic development. A medulloepithelioma can thus arise from any point along this pathway.

The presentation of an optic nerve medulloepithelioma depends on its location. When the tumour involves the orbital portion of the optic nerve, there is proptosis and optic disc swelling, similar to that produced by an optic 

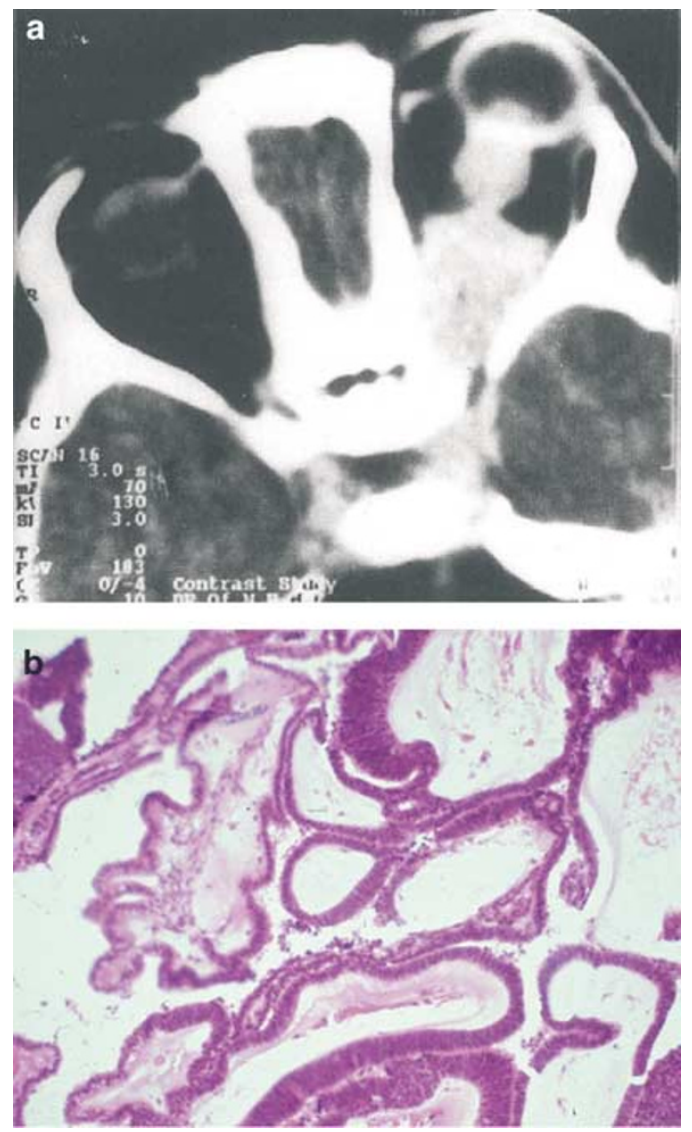

Figure 7 Medulloepithelioma of the optic nerve: (a) CT appearance; (b) histopathology (Courtesy of Dr W. Richard Green).

nerve glioma. More posterior lesions produce a progressive retrobulbar optic neuropathy. The imaging appearance of these lesions may mimic that of an optic nerve glioma, consisting of a fusiform enlargement of the nerve (Figure 7a).

Like its intracranial counterpart, the optic nerve medulloepithelioma is composed of multilaminar columnar cells that grow in tubes and cords, creating an elaborate interconnecting set of cellular strands that give the impression of a net (Figure 7b). Mitoses may be rare or abundant, and the degree of cellular anaplasia varies considerably among tumours. A simple

medulloepithelioma contains only elements that resemble medullary epithelium and structures derived from the optic vesicle, including retinal pigment epithelium, ciliary epithelium, vitreous, and neuroglia. There are also benign and malignant teratoid variants that contain one or more heteroplastic elements such as cartilage or striated muscle.

The treatment of an optic nerve medulloepithelioma is complete excision of the nerve; however, recurrences can occur despite gross total removal of the lesion, and metastatic spread may occur.
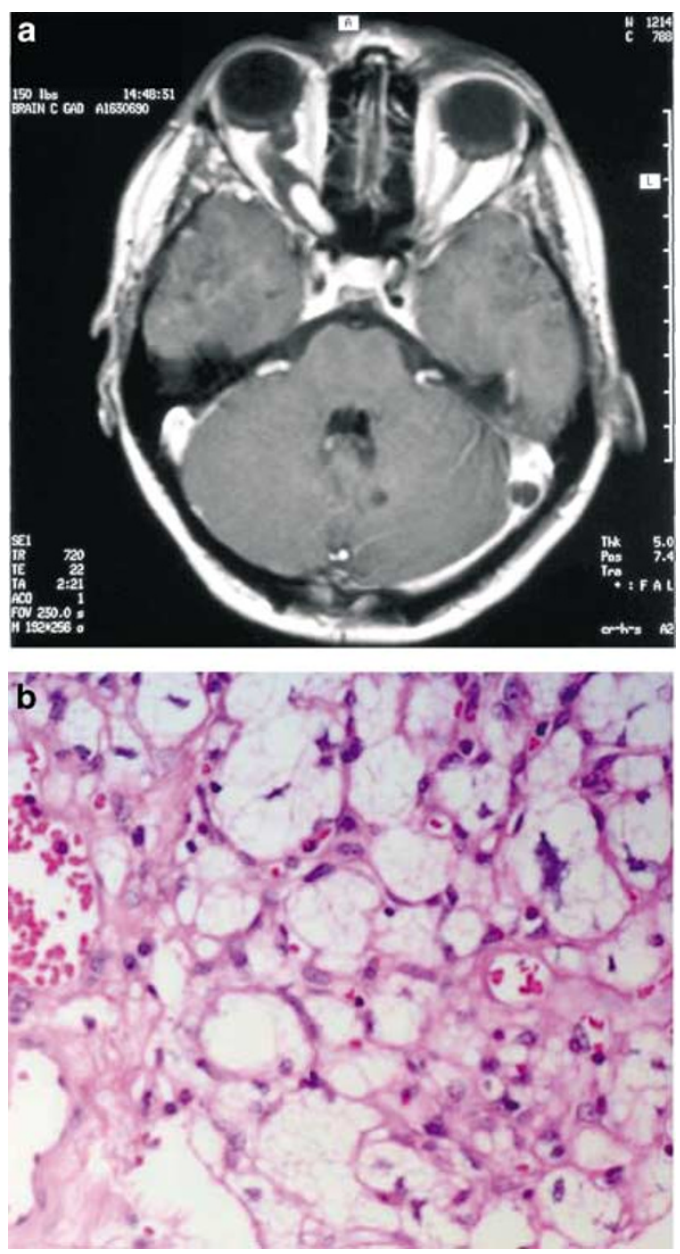

Figure 8 Haemangioblastoma of the optic nerve: (a) CT appearance; (b) histopathology. (Courtesy of Dr John Carter).

\section{Haemangioblastoma}

Haemangioblastomas occasionally occur within the substance of the optic nerve. ${ }^{23}$ The neuroimaging appearance of such lesions is similar to that of optic nerve gliomas (Figure 8a); however, they generally show much more enhancement which tends to be diffuse. Some these patients have evidence of von Hippel-Lindau disease. In other cases, the tumour appears to be an isolated phenomenon.

Haemangioblastomas are composed of two principal cellular components. One is the endothelial cell with accompanying pericytes. The other is an interstitial or stromal cell. The ratio of these two elements, the caliber of the interposed vascular channels and the degree of lipidization of stromal cells, contribute to the histologic heterogeneity of the tumour (Figure $8 \mathrm{~b}$ ).

Although the name 'hemangioblastoma' implies a poorly differentiated lesion with malignant or at least invasive characteristics, haemangioblastomas are benign 
lesions with little tendency to seed, metastasize, or recur once they are removed. Their treatment is total removal whenever possible. This can usually be accomplished through adequate exposure of the tumour at surgery, microsurgical technique, and careful attention to haemostasis.

\section{Tumours of the optic nerve sheath}

The most common tumour of the optic nerve sheath is the meningioma; however, a few rare tumours of the sheath have also been described, including one case of an haemangiopericytoma and several cases of schwannomas.

\section{Optic nerve sheath meningioma}

Optic nerve sheath meningiomas are usually separated into two types. ${ }^{24}$ Primary meningiomas are those that arise from the cap cells of the arachnoid surrounding the intraorbital or, less commonly, the intracanalicular portions of the optic nerve. Secondary meningiomas arise intracranially, usually from the sphenoid ridge, tuberculum sellae or olfactory groove and subsequently invade the optic canal and orbit by extending between the dura and arachnoid of the optic nerve in these regions. The term 'optic nerve sheath meningioma' thus does not connote a definitive site of origin. Nevertheless, once this type of tumour gains access to the subdural space of the intracanalicular or intraorbital optic nerve, the tumour grows up or down the sheath, invading the dura and obliterating the pial blood supply. In some instances, the tumour encircles the optic nerve without invading it. In other cases, the tumour invades the nerve by growing along the fibrovascular pial septa. Such a tumour may eventually surround and obstruct the central retinal vein, central retinal artery, or both vessels. Rarely, a tumour will break through the dural sheath of the optic nerve to invade other orbital structures. Finally, a tumour that originates within the optic nerve sheath in the orbit or optic canal may grow intracranially to involve the optic chiasm, contralateral optic nerve, and internal carotid artery. It may even invade the cavernous sinus or the sella turcica.

The symptoms and signs of optic nerve sheath meningiomas depend in large part on whether they have arisen within the orbit, within the optic canal, or intracranially.

Primary optic nerve sheath meningioma (perioptic meningioma)

As noted above, these tumours arise from cap cells of the arachnoid surrounding the optic nerve in the orbit or optic canal. Their histology is that of syncytial or transitional meningiomas.

As. with other meningiomas, primary optic nerve sheath meningiomas most often develop in middle-aged women but may occur in adult men and also in children. ${ }^{25}$ Most of these tumours are unilateral, but bilateral cases do occur, most often in adolescent men and women.

Patients with optic nerve sheath meningiomas usually complain initially of decreased or blurred vision in their affected eye, although at the time of examination, the vision may be $20 / 20$ or better. According to Dutton, $97 \%$ have visual loss at the time of presentation but $45 \%$ have vision of $20 / 40$ or better and less than $25 \%$ have count fingers vision or worse. ${ }^{25}$ In most of these patients, there is evidence of an underlying optic neuropathy (diminished colour vision or at least relative desaturation of red compared with the opposite eye; relative afferent pupillary defect), but this is not always the case. Other patients complain only of transient visual loss lasting a few seconds, similar to that described by some patients with increased increased intracranial pressure and papilloedema. In some of these cases, the visual loss occurs only when moving the eye into a particular field of gaze. ${ }^{26,27}$

The prevalence of proptosis and strabismus vary in different series but are rarely initial symptoms and usually occur after visual loss has begun. The proptosis usually varies from 2 to $5 \mathrm{~mm}$. Motility abnormalities are present in at least half of the patients with these lesions but most patients are asymptomatic.

In patients with primary optic nerve sheath meningiomas, the optic disc of the affected eye may be swollen, pale, or normal in appearance. The optic disc is usually swollen when the tumour surrounds or compresses the intraorbital portion of the nerve, and in this setting, there is rarely, if ever, any peripapillary haemorrhage (Figure 9a). When the patient has visual complaints and evidence of an underlying optic neuropathy, the diagnosis is rarely in question; however, in some cases, the patient's examination is relatively unremarkable. Such patients may be thought to have unilateral 'papilloedema' if their visual symptoms (ie, blurred or foggy vision) are ignored by their physician. They may thus be evaluated not for an orbital process but for increased intracranial pressure.

Although many patients with optic nerve sheath meningiomas involving the orbital portion of the optic nerve initially have relatively good visual function, with time, vision gradually is lost. As this occurs, the patient's optic disc swelling begins to resolve, and retinochoroidal (optociliary shunt) vessels may appear on the surface of the disc (Figure 9b). These vessels shunt venous blood from the retinal to the choroidal circulation, thereby 

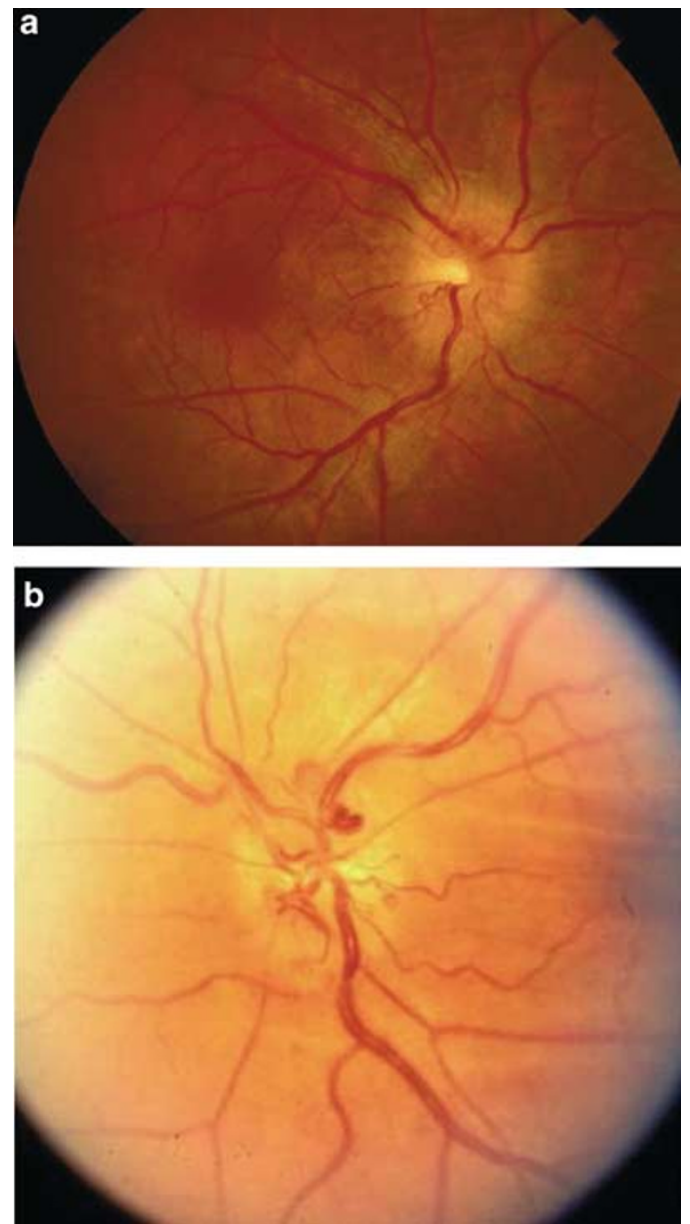

Figure 9 Optic nerve sheath meningioma (primary): (a) swollen optic disc. Note the absence of hemorrhage; (b) retinochoroidal vessels.

allowing egress via the superior or inferior ophthalmic veins and bypassing of the central retinal vein. ${ }^{28}$ When optociliary shunt vessels are acquired, they are virtually pathognomonic of chronic central retinal vein compression, and the triad of visual loss, optic atrophy, and optociliary shunt veins is most commonly caused by an optic nerve sheath meningioma. Nevertheless, retinochoroidal shunt vessels are seen in only a third of optic nerve meningiomas. This may be in part because they often become more subtle or resolve as atrophy progresses. $^{25}$

Not all patients with primary optic nerve sheath meningiomas have swollen optic discs. When the tumour originates at the apex of the orbit or within the optic canal, there is slowly progressive visual loss without orbital signs, usually with a normal-appearing optic disc. Although such patients almost always have evidence of an underlying optic neuropathy, this may be overlooked by the examining physician. With time, the disc becomes
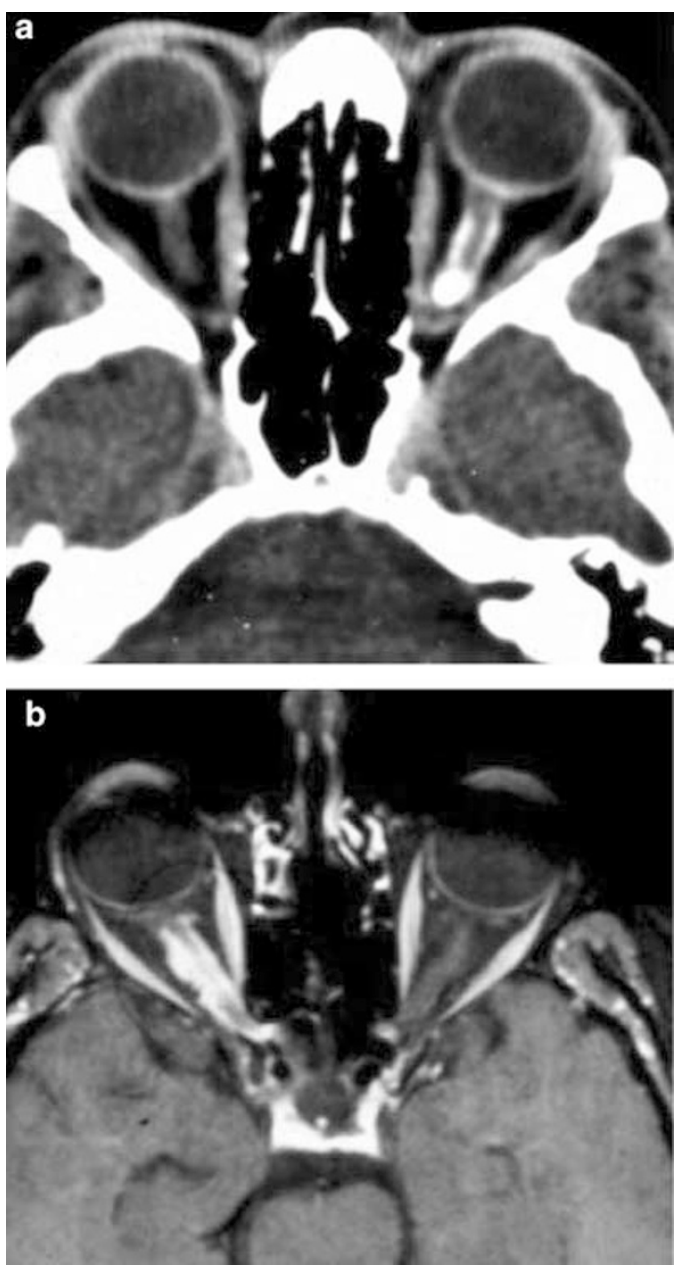

Figure 10 Neuroimaging appearance of optic nerve sheath meningioma: (a) CT scan; (b) MR imaging.

pale, but until it does, the true cause of the visual loss may be obscure.

Once the diagnosis of a primary optic nerve sheath meningioma is suspected, the diagnosis can usually be established using high-resolution CT scanning or MR imaging (Figure 10). ${ }^{25,29}$ The characteristic features of optic nerve sheath meningiomas include the presence of calcification surrounding the nerve (which occurs in 20-50\%), tubular enlargement of the nerve, and a bulbous enlargement of the optic nerve at the apex with distal tubular enlargement. The appearance of the optic nerve on coronal section is a hypodense area surrounded by a dense peripheral ring. Marked, homogenous enhancement with gadolinium is also typical. On very rare occasions, small tumours located within the optic canal remain impossible to detect using current neuroimaging procedures. Such lesions are discovered only during exploratory craniotomy. The lesions may be suspected, however, in any patient with slowly 
progressive, unilateral loss of vision associated with signs of optic neuropathy. In addition, the presence of enlarged, aerated posterior ethmoid and sphenoid sinuses, a condition known as pneumosinus dilatans, is thought by some authors to be pathognomonic of a meningioma. ${ }^{30}$

The treatment of primary optic nerve sheath meningiomas has changed dramatically in the last few years. These lesions are compatible with good vision for many years, are not life threatening, and are unlikely to spread intracranially to the extent that they produce neurologic dysfunction. ${ }^{31}$ In addition, although some of these lesions spread across the planum sphenoidale to enter the contralateral optic canal and compress the opposite optic nerve, even this type of extension is unusual. Thus, the main threat of this tumour is eventual unilateral blindness, which may take years or even decades. In addition, optic nerve sheath meningiomas cannot be separated from the optic nerve because, in most cases, the tumour completely surrounds (and occasionally invades) the optic nerve and is so diffuse that it cannot be peeled away, and during the attempt to remove such lesions, there may be damage to the pial vasculature resulting in loss of remaining vision. Thus, in the past, the options were limited to surgical removal of the affected optic nerve, resulting in blindness in the affected eye, or following the patient without intervention, in which case the patient slowly lost vision in the eye. Most physicians and patients chose the latter alternative. More recently, it has become clear that surgery to remove an optic nerve sheath meningioma (along with the affected optic nerve) is rarely if ever indicated and that radiation is the optimum therapy. ${ }^{32,33}$ To date, the only long-term data reflect the results after treatment using conventional fractionated radiation therapy; ${ }^{34}$ however, there is increasing evidence that stereotactic or conformal fractionated radiation therapy may result in improved visual outcome with fewer complications..$^{29,35-37}$ I recommend that patients with optic nerve sheath meningiomas who have good visual function be followed without intervention until or unless they begin to experience progressive loss of vision, at which time I recommend stereotactic or conformal fractionated radiation therapy, whereas patients who present with significant visual loss should be given the option to undergo radiation therapy immediately. In such patients, the progression of visual loss may be extremely slow, occurring over many years. Such patients should undergo regular clinical examinations every 3-6 months and repeat CT scanning or MR imaging every 6-12 months.

When there is evidence of significant intracranial spread of tumour across the planum sphenoidale in a patient with a primary optic nerve sheath meningioma and useful visual function, a craniotomy should be considered to remove the intracranial portion of tumour, thereby preventing tumour spread to the contralateral optic nerve. In such cases, the tumour surrounding the intracanalicular and orbital portions of the affected optic nerve is usually left alone, although the optic canal may be opened and the sheath split.

\section{Secondary optic nerve sheath meningioma}

These tumours originate intracranially, usually in the region of the planum sphenoidale or tuberculum sellae and gradually extend into the optic canal. In many cases, it may be difficult, even at surgery, to determine whether a meningioma began in the posterior orbit or optic canal and then spread intracranially, or whether the tumour began intracranially and spread into the optic canal. In both cases, there is slowly progressive visual loss associated with evidence of an optic neuropathy and a normal or pale optic disc. Neuroimaging should be performed to identify and follow these lesions.

The problems inherent to treating secondary optic nerve sheath meningiomas are similar to those that relate to primary optic nerve sheath meningiomas. If there is a substantial intracranial component, surgery should be considered to prevent the tumour from involving other crucial intracranial structures, including the opposite optic nerve, optic chiasm, internal carotid artery, pituitary gland, and cavernous sinus. If residual tumour remains, postoperative radiation therapy should be considered to minimize progression and recurrence.

\section{Schwannoma}

Schwannomas are benign tumours that arise from Schwann cells in the peripheral nervous system. Although the most common sites are the vestibular division of the VIIIth cranial nerve and the trigeminal nerve root, schwannomas occasionally involve the optic nerve. ${ }^{38-40}$ As optic nerve myelin is produced by oligodendrocytes rather than Schwann cells, these tumours probably arise from the Schwann cells that accompany the sympathetic nerves that are tightly adherent to the optic nerve sheath. ${ }^{41}$

The microscopic appearance of schwannomas of the optic nerve is the same as for all schwannomas of peripheral nerve origin, thus distinguishing them from similar appearing lesions, such as optic nerve gliomas or meningiomas; however, the clinical presentation of these lesions is nonspecific. They have been reported in both children and adults, all of whom have developed progressive visual loss associated with evidence of an optic neuropathy and variable proptosis. 

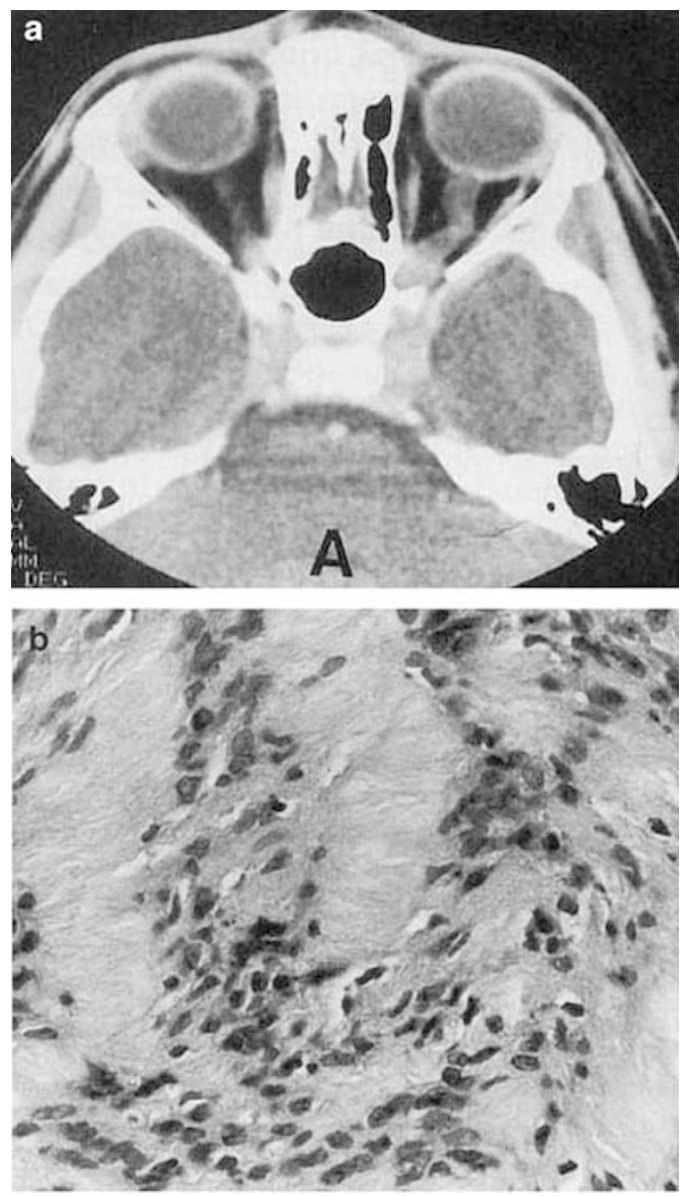

Figure 11 Optic nerve sheath schwannoma: (a) CT appearance; (b) histopathology.

Optic nerve sheath schwannomas probably cannot be diagnosed on clinical grounds alone, and their neuroimaging appearance mimics that of the more common optic nerve gliomas (Figure 11). Instead, in the reported cases, the diagnosis has been made at surgery. To date, surgery has been the treatment of choice, but in view of the increasing tendency to treat many intracranial schwannomas with stereotactic radiosurgery, this treatment option perhaps should be considered for schwannomas of the optic nerve.

\section{Haemangiopericytoma}

Haemangiopericytomas are composed of the pericytes of blood vessels (Figure 12). They may develop in virtually any tissue in the body that has capillaries. It was once thought that haemangiopericytomas were actually a type of angioblastic meningioma that arose from the vascular elements of the pia mater. Ultrastructural studies, however, have led to a better understanding of the
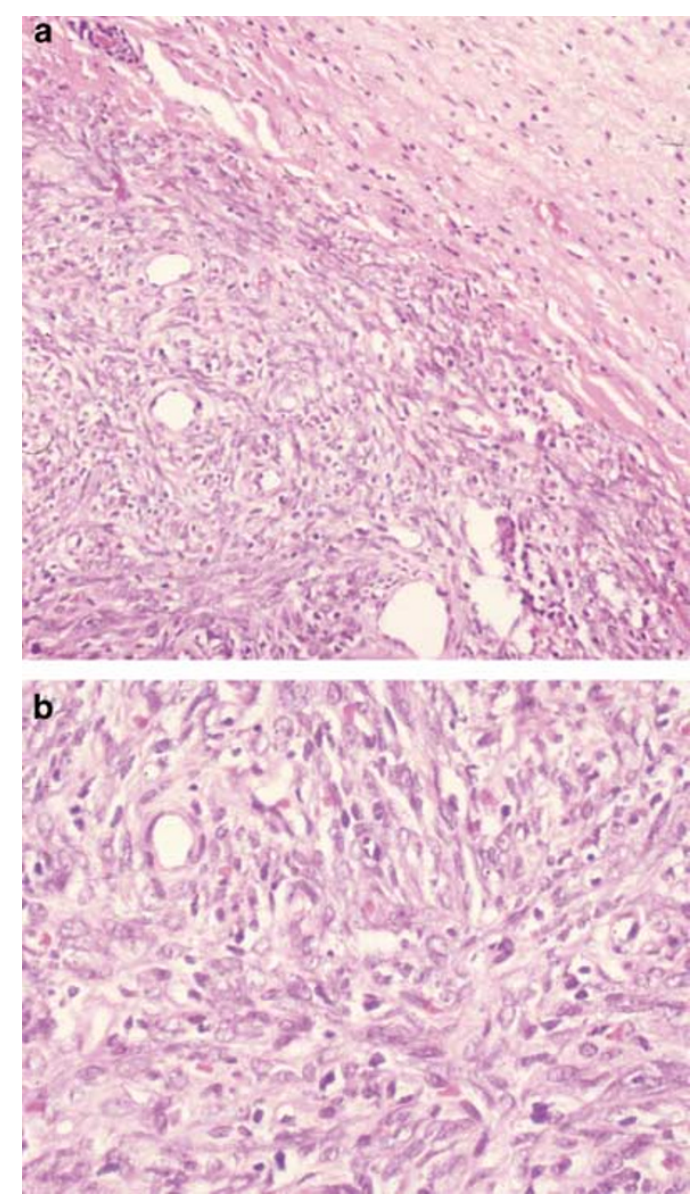

Figure 12 Histopathology of optic nerve sheath haemangiopericytoma: (a) relationship of tumour to dural sheath; (b) higher power of tumour cells (Courtesy of Dr W Richard Green).

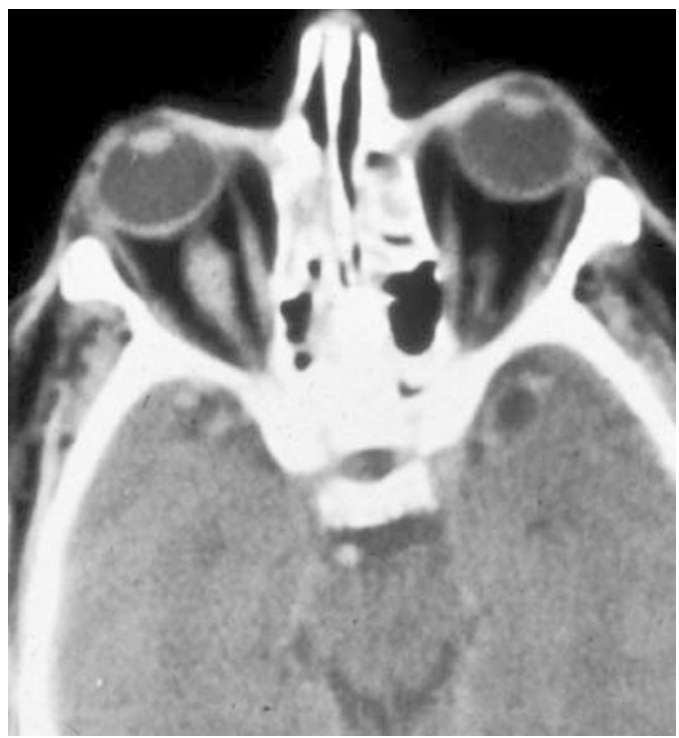

Figure 13 CT appearance of an optic nerve sheath haemangiopericytoma (Courtesy of Dr RL Font). 
histogenesis of these tumours. By light microscopy, haemangiopericytomas are composed of a monomorphous proliferation of plump or spindleshaped cells. Ultrastructurally, the pericytes show varying degrees of differentiation into smooth muscle cells, glomus cells, endothelial cells, and fibroblasts.

Haemangiopericytomas, unlike meningiomas, have no sex predilection. They usually become symptomatic in the fifth decade of life, usually are painless and enlarge slowly.

I am aware of a single case of a haemangiopericytoma of the optic nerve sheath. ${ }^{42}$ The patient was a 61 -year-old man who experienced progressive visual loss in the right eye following a systemic viral illness. He was thought to have optic neuritis but the visual loss worsened considerably of the next few months and a CT scan showed a focal fusiform enlargment of the orbital portion of the right optic nerve thought to be consistent with an optic nerve sheath meningioma (Figure 13). The patient subsequently underwent exploration of the right orbit at which time the abnormal portion of the optic nerve was excised. Pathologic examination of the specimen revealed an intradural haemangiopericytoma compressing an otherwise normal optic nerve.

The treatment of choice for haemangiopericytomas is complete surgical excision. The roles of both radiation therapy and chemotherapy are controversial.

\section{Summary}

Most tumours of the optic nerve and its sheath are benign and produce slowly progressive visual loss associated with evidence of an anterior or posterior optic neuropathy and variable proptosis. The diagnosis of the most common tumours - gliomas and menintioma - can be made with neuroimaging. However, in some cases, the diagnosis is not made until the orbit is explored and a biopsy of the nerve is obtained or the affected nerve is excised. The treatment of optic nerve tumours depends on the nature of the lesion. In some cases, excision of the nerve is appropriate; however, in most, observation, radiation, or chemotherapy is a better choice. The visual prognosis of optic nerve tumours depends on the nature of the lesion.

\section{References}

1 Dutton JJ. Gliomas of the anterior visual pathway. Surv Ophthalmol 1994; 38: 427-452.

2 Chutorian AM, Schwartz JF, Evans RA, Carter S. Optic gliomas in children. Neurology 1964; 14: 83-95.

3 Rush JA, Younge BR, Campbell RJ, MacCarty CS. Optic glioma: long-term follow-up of 85 histologically verified cases. Ophthalmology 1982; 89: 1213-1219.
4 Tow SL, Chandela S, Miller NR, Avellino AM. Long-term prognosis in children with gliomas of the anterior visual pathway. Pediatr Neurol 2003; 28: 262-270.

5 Sharma A, Mohan K, Saini JS. Haemorrhagic changes in pilocytic astrocytoma of the optic nerve. Orbit 1990; 9: 29-33.

6 Listernick R, Louis DN, Packer RJ, Gutmann DH. Optic pathway gliomas in children with neurofibromatosis type 1 : consensus statement from the NF1 Optic Glioma Task Force. Ann Neurol 1997; 41: 143-149.

7 Brodsky MC. The 'pseudo-CSF' signal of orbital optic gliomas on magnetic resonance imaging: a signature of neurofibromatosis. Surv Ophthalmol 1993; 38: 213-218.

8 Cummings TJ, Provenzale JM, Hunter SB, Friedman AM, Klintworth GK, Bigner SH et al. Gliomas of the optic nerve: histological, immunohistochemical (MIB-1 and p53), and MRI analysis. Acta Neuropathol 2000; 99: 563-570.

9 Liauw L, Vielvoye GJ, de Keizer JR, van Duinen SG. Optic nerve glioma mimicking an optic nerve meningioma. Clin Neurol Neurosurg 1996; 98: 258-261.

10 Stern J, Jakobiec FA, Housepian EM. The architecture of optic nerve gliomas with and without neurofibromatosis. Arch Ophthalmol 1980; 98: 505-511.

11 Parsa CF, Hoyt WF, Lesser RL, Weinstein JM, Strother GM, Muci-Mendoza R et al. Spontaneous regression of optic gliomas. thirteen cases documented by serial neuroimaging. Arch Ophthalmol 2001; 119: 516-529.

12 Lee AG, Dutton JJ. A practice pathway for the management of gliomas of the anterior visual pathway: an update and an evidence-based approach. Neuro-ophthalmology 1999; 22: 139-155.

13 Kortmann R-D, Timmermann B, Paulsen F, Kay S, Schedierbauer J, Plasswilm L et al. The role of radiotherapy in the management of low grade glioma of the visual pathway in children. Neuro-ophthalmology 2002; 27: 17-37.

14 Spoor TC, Kennerdell JS, Martinez Z, Zorub D. Malignant gliomas of the optic nerve pathways. Am J Ophthalmol 1980; 89: 284-292.

15 Brodovsky S, ten Hove MW, Pinkerton RM, Ludwin SK, Smith RM. An enhancing optic nerve lesion: malignant glioma of adulthood. Can J Ophthalmol 1997; 32: 409-413.

16 Taphoorn MJB, de Vries-Knoppert WAEJ, Ponssen $\mathrm{H}$, Wolbers JG. Malignant optic glioma in adults. Case report. J Neurosurg 1989; 70: 277-279.

17 Gritzman MCD, Snyckers FD, Proctor NS. Ganglioglioma of the optic nerve. A case report. S Afr Med J 1983; 63: 863-865.

18 Bergin DJ, Johnson TE, Spencer WH, McCord CD. Ganglioglioma of the optic nerve. Am J Ophthalmol 1988; 105: $146-149$.

19 Sadun F, Hinton DR, Sadun AA. Rapid growth of an optic nerve ganglioglioma in a patient with neurofibromatosis 1 . Ophthalmology 1996; 103: 794-799.

20 Green WR, Iliff WJ, Trotter RA. Malignant teratoid medulloepithelioma of the optic nerve. Arch Ophthalmol 1974; 91: 451-454.

21 Chidambaram B, Santosh V, Balasubramanian V. Medulloepithelioma of the optic nerve with intradural extension-report of two cases and a review of the literature. Child's Nerv System 2000; 16: 329-333.

22 Chavez M, Mafee MF, Castillo B, Kaufman LM, Johnstone $\mathrm{H}$, Edward DP. Medulloepithelioma of the optic nerve. J Pediatr Ophthalmol Strabismus 2004; 41: 48-52. 
23 Kerr DJ, Scheithauer BW, Miller GM, Ebersold MJ, McPhee TJ. Hemangioblastoma of the optic nerve: case report. Neurosurgery 1995; 36: 573-581.

24 Cockerham KP, Kennerdell JS, Maroon JC. Tumours of the meninges and related tissues: meningiomas and sarcomas. In: Miller NR, Newman NJ (eds) Walsh and Hoyt's Clinical Neuro-Ophthalmology, 5th ed., Vol 2. Williams \& Wilkins: Baltimore, 1998, pp 2017-2082.

25 Dutton JJ. Optic nerve gliomas and meningiomas. Neurol Clin 1991; 9: 163-177.

26 Bradbury PG, Levy IS, McDonald WI. Transient uniocular visual loss on deviation of the eye in association with intraorbital tumours. J Neurol Neurosurg Psychiatry 1987; 50: 615-619.

27 Oohira A, Kubo R. Ocular blood flow defect in gaze-evoked amaurosis. J Jpn Ophthalmol Soc 1999; 103: 56-60.

28 Muci-Mendoza R, Arevalo JF, Ramella M, FuenmayorRivera D, Karam E, Cardenas PL et al. Optociliary veins in optic nerve sheath meningioma: indocyanine green videoangiography findings. Ophthalmology 1999; 106: 311-318.

29 Andrews DW, Faroozan R, Yang BP, Hudes RS, WeinerWasik M, Kim SM et al. Fractionated stereotactic radiotherapy for the treatment of optic nerve sheath meningiomas: preliminary observations of 33 optic nerves in 30 patients with historical comparison to observation with or without prior surgery. Neurosurgery 2002; 51: 890-904.

30 Hirst LW, Miller NRM, Hodges III FJ, Corbett JJ, Thompson HS. Sphenoid pneumosinus dilatans: A sign of meningioma originating in the optic canal. Neuroradiology 1982; 22: 207-210.

31 Egan RA, Lessell S. A contribution to the natural history of optic nerve sheath meningiomas. Arch Ophthalmol 2002; 120: 1505-1508.

32 Miller NR. Radiation for optic nerve meningiomas: is this the answer? Ophthalmology 2002; 109: 833-834.
33 Miller NR. The evolving management of optic nerve sheath meningiomas. Br J Ophthalmol 2002; 86: 1198.

34 Turbin RE, Thompson CR, Kennerdell JS, Cockerham KP, Kupersmith MJ. A long-term outcome comparison in patients with optic nerve sheath meningioma managed with observation, surgery, radiotherapy, or surgery and radiotherapy. Ophthalmology 2002; 109: 890-900.

35 Lee AG, Woo SY, Miller NR, Safran AB, Grant WH, Butler EB. Improvement in visual function in an eye with a presumed optic nerve sheath meningioma after treatment with three-dimensional conformal radiation therapy. J Neuroophthalmol 1996; 16: 247-251.

36 Liu JK, Forman S, Hershewe GL, Moorthy CR, Benzil DL. Optic nerve sheath meningiomas: visual improvement after stereotactic radiotherapy. Neurosurgery 2002; 50: 950-957.

37 Pitz S, Becker G, Schiefer U, Wilhelm H, Jeremic B, Bamberg $\mathrm{M}$ et al. Stereotactic fractionated irradiation of optic nerve sheath meningioma: a new treatment alternative. $\mathrm{Br} \mathrm{J}$ Ophthalmol 2002; 86: 1265-1268.

38 Simpson RK, Harper RL, Kirkpatrick JB, Cooper B. Schwannoma of the optic sheath. J Neuroophthalmol 1987; 7: 219-222.

39 Saini JS, Mohan K, Sharma A. Pirmary orbital optic nerve sheath schwannoma. Orbit 1990; 9: 97-99.

40 Kim D-S, Choi J-U, Yang K-H, Jung J-M. Optic sheath schwannomas: report of two cases. Childs Nerv Syst 2002; 18: 684-689.

41 Anderson DR, Hoyt WF. Ultrastructure of intraorbital portion of human and monkey optic nerve. Arch Ophthalmol 1969; 82: 506-530.

42 Boniuk M, Messmer EP, Font RL. Hemangiopericytoma of the meninges of the optic nerve. A clinicopathologic report including electron microscopic observations. Ophthalmology 1985; 92: 1780-1787. 